\title{
Correlation between Temporomandibular Joints and Nasal Cavity Width in Growing Patients after Rapid Maxillary Expansion
}

\author{
Mona S Ghoussoub ${ }^{1}$, Ghassan Sleilaty ${ }^{2}$, Robert Garcia ${ }^{3}$, Khaldoun Rifai ${ }^{4}$
}

\begin{abstract}
Aim: The study tests the correlation between the enlargement of the nasal cavity width, interglenoid fossa distance, and intercondylar distance after rapid maxillary expansion (RME) in growing patients.

Materials and methods: Cone beam computed tomography (CBCT) was performed for 25 patients presenting a bilateral crossbite ( 11 males, 14 females, and mean age $11.6 \pm 1.6$ years) at baseline (T0) and at 6 months after RME (T2), T1 being the end of expander activation. Images were digitized for linear measurements using specific software. Values were compared at the nasal width, interglenoid fossa distance, and intercondylar distance to test the correlation in the transverse dimension.

Results: At T0, a correlation already existing between the interglenoid fossa distance and the intercondylar distance persisted at T2. The correlation between the nasal cavity width and interglenoid fossa distance nonexistent at T0 ended toward statistically significant at T2. Additionally, the lateral position of the condyles was not correlated with the nasal cavity width neither at T0 or T2.

Conclusion: A correlation between the interglenoid fossa distance and intercondylar distance was exhibited 6 months after RME. The correlation between the nasal cavity width and interglenoid fossa distance was close to significant at T2.

Keywords: Cone beam computed tomography, Correlation, Intercondylar distance, Interglenoid fossa distance, Nasal cavity width.

The Journal of Contemporary Dental Practice (2019): 10.5005/jp-journals-10024-2580
\end{abstract}

\section{INTRODUCTION}

The earliest report of maxillary expansion was in 1860 by Emerson Colon Angell, who claimed to have achieved opening of the midpalatal suture and described a gap between the maxillary central incisors. ${ }^{1-3}$ Rapid maxillary expansion (RME) became commonly used only after the second half of the 20th century with the landmark study conducted by Haas., ${ }^{1,4}$

Mc Namara et al. conducted a research to evaluate papers on the changes in airway dimensions produced by RME in growing patients. The results demonstrated that widening of the nasal cavity base allows the reduction in nasal airway resistance with an improvement of the respiratory pattern. ${ }^{5}$

Few studies concerning the effect of RME on TMJs were conducted. Melgaço et al. carried out a study to test if RME promotes immediate changes in the position of the mandibular condyles in young patients with class I malocclusion. The results revealed statistically significant anterior and inferior displacements of these structures with lateral inclinations of both condyles in the coronal plane with no asymmetrical pattern. ${ }^{2}$ Another research performed by McLeod et al. aiming to explore radiologically the three-dimensional condylar modifications after RME in growing individuals concluded that therapies applying a tooth-anchored device do not produce relevant alterations of the condyle with respect to the glenoid fossa, thus not representing a limitation for engaging this procedure. ${ }^{6}$

This clinical trial aims to assess in young subjects, displaying a bilateral crossbite treated with RME, the correlation between the nasal cavity width, interglenoid fossa distance, and intercondylar distance to detect any asymmetrical alteration in the TMJs and to reveal the impact of this therapeutic procedure on both neighboring and distant structures from the site of expander activation.
${ }^{1}$ Department of Orthodontics, School of Dental Medicine, Hadath, Lebanon

${ }^{2}$ Department of Biostatistics, Faculty of Medicine, Saint Joseph University, Beirut, Lebanon

${ }^{3}$ School of Dental Medicine, Paris Diderot University (Paris VII), Paris, France

${ }^{4}$ Department of Prosthodontics, School of Dental Medicine, Hadath, Lebanon

Corresponding Author: Mona S Ghoussoub, Department of Orthodontics, School of Dental Medicine, Hadath, Lebanon, Phone: +961 3559095, e-mail: mona_gsoub@yahoo.fr

How to cite this article: Ghoussoub MS, Sleilaty G, et al. Correlation between Temporomandibular Joints and Nasal Cavity Width in Growing Patients after Rapid Maxillary Expansion. J Contemp Dent Pract 2019;20(6):686-692.

Source of support: The protocol was approved by the Ethics Committee of the Lebanese University, National Institute in Lebanon [CUEMB process number 31/04/2015]. The study was funded by the Lebanese University and Centre National de Recherche Scientifique, Lebanon (CNRS) [Number: 652 on 14/04/2016]. The CNRS has no role in the design of the study, neither collection, analysis, nor interpretation of data or in writing the manuscript.

Conflict of interest: None

Trial registration: Retrospectively registered in International Standard Registered Clinical/Soial Study Number (ISRCTN) (DOI: 10.1186/ ISRCTN77788053).

\section{Materials and Methods}

The protocol of the current clinical trial was agreed by the Ethics Committee of the Lebanese University, Hadath, Lebanon

(0) The Author(s). 2019 Open Access This article is distributed under the terms of the Creative Commons Attribution 4.0 International License (https://creativecommons. org/licenses/by-nc/4.0/), which permits unrestricted use, distribution, and non-commercial reproduction in any medium, provided you give appropriate credit to the original author(s) and the source, provide a link to the Creative Commons license, and indicate if changes were made. The Creative Commons Public Domain Dedication waiver (http://creativecommons.org/publicdomain/zero/1.0/) applies to the data made available in this article, unless otherwise stated. 
(registration number: CUEMB 31/4/2015). Registration was performed retrospectively in ISRCTN registry (DOI: 10.1186/ ISRCTN77788053). ${ }^{7}$ Children's parents or guardians approved and signed the consent form. The screening campaign was achieved during 2016, and recruitment was started in September 2016. The study was performed at the Department of Orthodontics of the Lebanese University School of Dentistry, Hadath, Lebanon. Patients presenting bilateral crossbite were enrolled in the orthodontic unit and in seven neighboring schools. A general diagnosis concerning the medical and dental history was performed prior to radiological and therapeutic procedures. Inclusion and exclusion criteria following standardized examination forms are entailing the succeeding

Inclusion criteria:

- Patients between 8 years and 13 years old;

- Revealing a maxillary skeletal deficiency in the transverse dimension, with bilateral crossbite including one or more lateral teeth;

- Exposing a narrow tapered palatal vault;

- Presenting tooth size-arch length discrepancy;

- Exhibiting an appropriate crown height (3-4 mm) for enough adaptability of the RME apparatus.

Exclusion criteria:

- Craniofacial syndromes;

- Presence of periodontal disease;

- Missing maxillary posterior permanent teeth (first molars);

- Prior orthodontic treatment.

At baseline (T0), cone beam computed tomography (CBCT) was seized using an $\mathrm{iCat}{ }^{\circledR}$ machine (Imaging Sciences International, Hatfield, PA) and at 6 months after the end of expansion activation (T2) with respect to a prevailing radiographic protocol (120 kVp; $5 \mathrm{~mA}$; field of view, $13 \times 17 \mathrm{~cm}$; voxel $0.4 \mathrm{~mm}$; scan time, 20 seconds). At T1, 3 weeks from baseline, matching with the end of expander activation, the opening of the midpalatal suture was appraised clinically by the appearance of a central diastema between incisors. The patients were scanned having the head positioned with Frankfort and midsagittal planes, respectively, parallel and perpendicular to the ground. They stayed in maximum intercuspation during the radiological capture of images. Data were reconstructed with $0.4 \mathrm{~mm}$ slice thickness and saved as digital imaging and communications in medicine (DICOM) files.

The same laboratory fabricated the expanders using screws of $10 \mathrm{~mm}$ length (Leone S.p.a., Italy-Ref. A2620-10). The procedure entailed that screws can be activated twice per day $(0.25 \mathrm{~mm}$ per turn) the first week, after that once per day for the following week, and every other day for the third week. The expander was kept in place to maintain the remodeling at the sutural site for a period of 6 months.

Construction of 3D surface models of the anatomic structures of interest was achieved using specific software Viewbox 4 [dHAL software by Demetrios Halazonetis, designer of the program, Kifissia, Greece; www.dhal.com]. Reconstruction with $0.5 \mathrm{~mm}$ slice thickness was then implemented.

\section{Radiographic Landmarks and Reference Planes}

Two operators (the principal investigator Mona Sayegh Ghoussoub (MSG) and a resident in orthodontics at the Lebanese University) adjusted to locate 3D CBCT landmarks of this study and then independently on $\mathrm{CBCT}$ images not belonging to the project with an interval of 2 weeks. Reproducibility was confirmed to analyze the intra and interexaminer agreement. Then, they determined landmarks on five CBCT images at T0 and T2. The outcomes were assessed at 2-week period to test the reliability.

Three-dimensional identification of 13 bony landmarks was achieved by scrolling in coronal, axial, and sagittal to define the best radiological definition of:

- $\mathrm{Na}$ (nasion): the most anterior point of the frontonasal suture (Figs $1 \mathrm{~A}$ to $\mathrm{C}$ );

- ANS (anterior nasal spine): the most prominent point of the palatal plane (Figs $1 \mathrm{~A}$ to $\mathrm{C}$ );

- S (sella): the center of pituitary fossa on a lateral view; localized with respect to the shape of this structure whose midpoint is identified by the program (Fig. 1C);

- Po right and left (porion): the highest point of the external acoustic meatus (Fig. 1A);

- Or right and left (orbitale): the lowest point of the orbit (Figs $1 \mathrm{~A}$ and B);

- CC right and left (center of the mandibular condyle): the midpoint of the condyles (Figs $2 \mathrm{~A}$ and $\mathrm{B}$ );

- HG right and left (highest summit of the glenoid fossa): the most elevated point of this structure (Fig. 2B);

- NC right and left (nasal cavity): the outmost point of the nasal cavity on the coronal view (Figs 1D and 3);

Skeletal changes were verified by measuring distances between specific landmarks. Frankfort and midsagittal planes were chosen as reference planes and constructed by localizing three landmarks for each (Fig. 4):

- Frankfort horizontal plane linking Po right, Po left, and the middle of the section between Or right and Or left;

- Midsagittal plane joining $\mathrm{S}, \mathrm{Na}$, and ANS.

To compute the linear changes at T0 and T2, the results were transferred in comma-separated values (CSV) format.
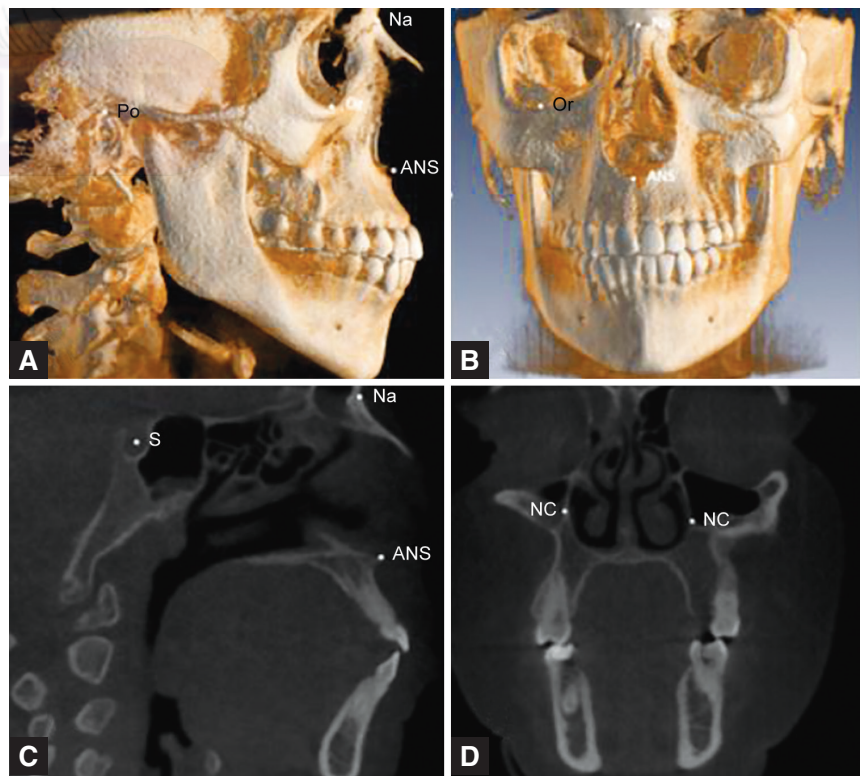

Figs 1 A to $D$ : (A to $D$ ) Three-dimensional identification of landmarks. (A to C) Nasion (Na); (A to C) Anterior Nasal Spine (ANS); (A and B) Orbitale (Or); (B) Porion (Po); (D) Nasal Cavity (NC) 

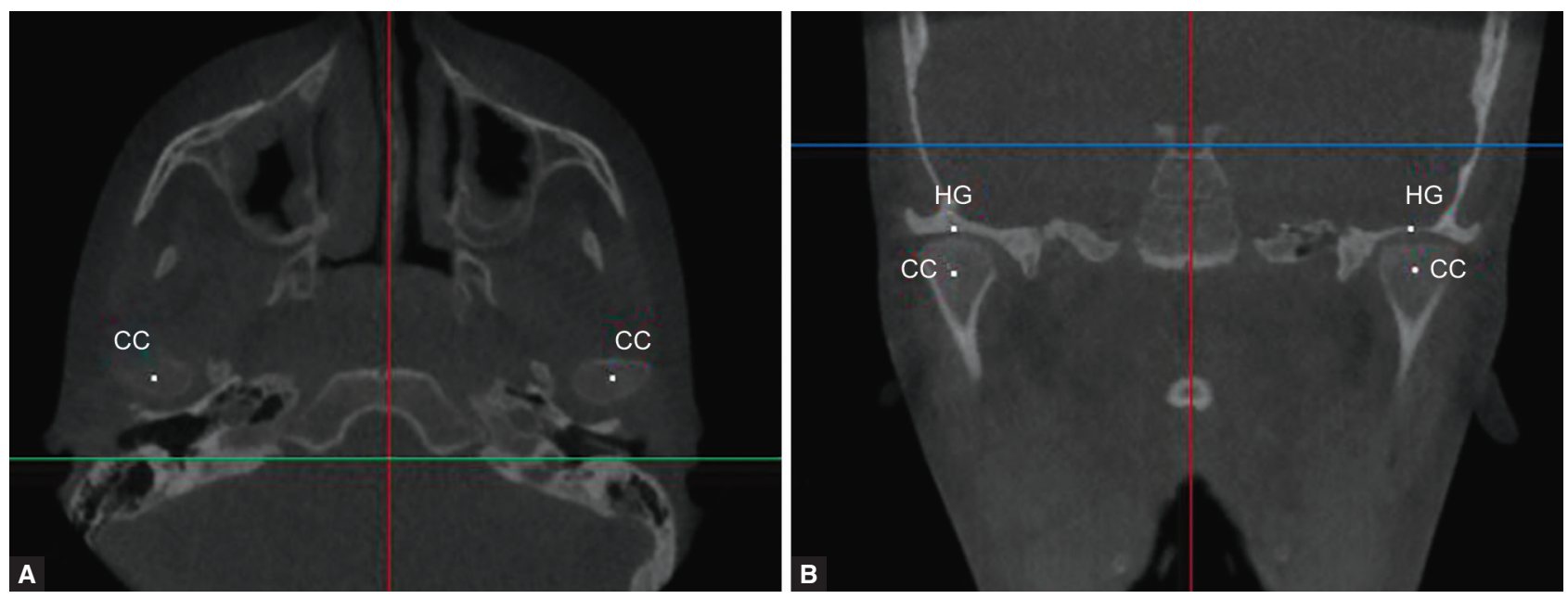

Figs $2 A$ and $B$ : (A and B) Center of the condyle (CC); (B) Highest point of the glenoid fossa (HG)

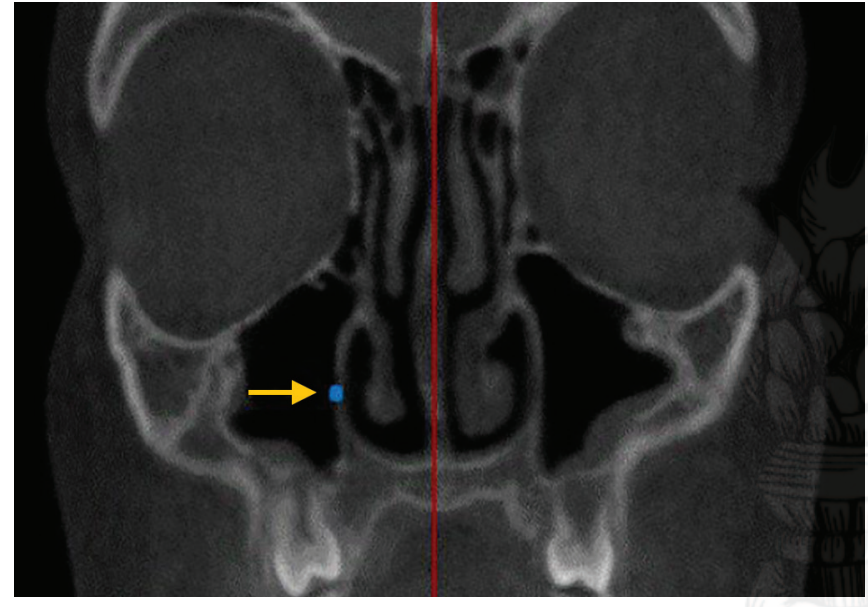

Fig. 3: Nasal cavity (NC) to the midsagittal plane

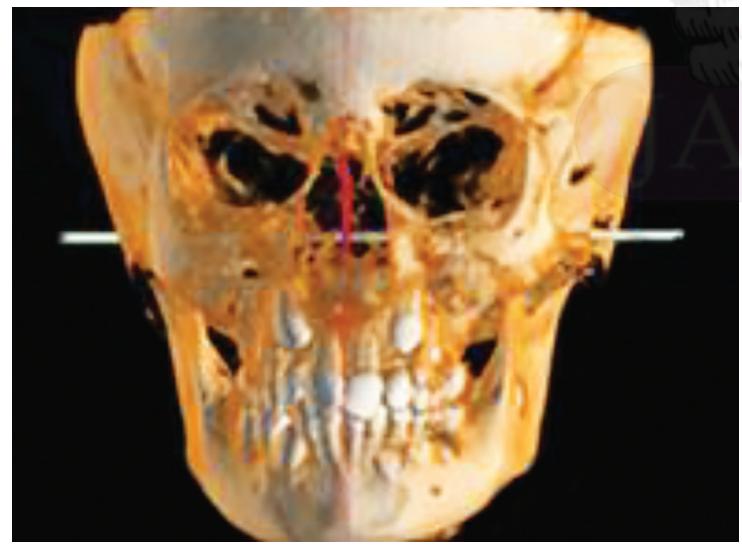

Fig. 4: Reference planes: the midsagittal plane in red and the Frankfort horizontal in light green

\section{Radiographic Parameters}

Radiographic changes in the interglenoid fossa and intercondylar distance as well as the transverse changes of the nasal width were evaluated in the transverse dimension. The following measures were noted for all the subjects on both sides at baseline and $\mathrm{T} 2$ following Melgaço et al.: ${ }^{2}$
- Measurement 1: the interglenoid fossa distance: evaluated on the frontal tomographic image calculating the distance between the uppermost point of the glenoid fossa and the midsagittal plane (HG_-midsagittal plane distance; right, left) (Fig. 2B).

- Measurement 2: the lateral location of the condyles: assessed on the axial tomographic image computing the segment between the center of the condyle and the midsagittal plane (CC-CC projected on the midsagittal plane; right, left) (Figs 2A and B).

- Measurement 3: the nasal cavity width: appraised on the frontal tomographic view evaluating the distance between the outmost point of the nasal aperture and the midsagittal plane (NC-midsagittal plane distance; right, left) (Fig. 3).

\section{Sample Size Calculation}

The current paper reports the correlation between the predefined parameters in the study group as defined in the study protocol in ISRCTN registry (DOI: 10.1186/ISRCTN77788053). ${ }^{7}$ There were 25 subjects who were included in the correlational analysis.

\section{Statistical Considerations}

Normality assumptions were checked graphically with quantilequantile plots and normality tests (Kolmogorov-Smirnov and Shapiro-Wilk). Bivariate Pearson's correlation coefficient was used to measure the correlation between the different $\mathrm{CBCT}$ quantitative measures at T0 and T2 (the lateral position of the glenoid fossa, the lateral position of the condyles, and the nasal width). The $95 \%$ confidence intervals of the correlation coefficients were derived by bootstrapping based on 1000 samples. The statistical analysis was performed using International Business Machines Statistical Package for the Social Sciences (IBM SPSS) (IBM Corp. Released 2013. IBM SPSS Statistics for Windows, Version 22.0. Armonk, NY).

\section{Results}

The study group included 25 subjects (11 males, 44.0\%; and 14 females, $56 \%$ ) aged $11.6 \pm 1.6$ years (range $7.4-14.2$ years). Baseline characteristics of the subjects are shown in Table 1 . All the predefined parameters satisfied normality distribution assumptions and were graphically checked with quantile-quantile plots.

Correlational analysis results at T0 and T2 are depicted in Table 2, showing the correlation coefficients, their associated $p$ values, and the corresponding $95 \%$ confidence intervals. 
Table 1: Baseline characteristics of the subjects with predefined parameters

\begin{tabular}{|c|c|c|c|c|c|c|}
\hline & & Count (\%) & Mean & Standard deviation & Minimum & Maximum \\
\hline$n$ & & 25 & & & & \\
\hline \multirow[t]{2}{*}{ Gender } & M & $11(44.0 \%)$ & & & & \\
\hline & $\mathrm{F}$ & $14(56.0 \%)$ & & & & \\
\hline \multicolumn{3}{|l|}{ Age } & 11.6 & 1.6 & 7.4 & 14.2 \\
\hline \multicolumn{3}{|c|}{$\begin{array}{l}\text { Lateral position of the glenoid } \\
\text { fossa @ T0 (mm) }\end{array}$} & 45.0 & 2.3 & 39.9 & 48.8 \\
\hline \multicolumn{3}{|c|}{$\begin{array}{l}\text { Laterolateral position of the } \\
\text { condyles @ T0 (mm) }\end{array}$} & 45.7 & 1.7 & 42.2 & 48.6 \\
\hline \multicolumn{3}{|c|}{ Nasal width @ T0 (mm) } & 28.3 & 2.1 & 25.3 & 34.5 \\
\hline
\end{tabular}

Table 2: Correlational analysis between the predefined CBCT parameters showing the statistically high significant correlation between the lateral position of the glenoid fossa and the lateral position of the condyles at T0 and T2

\begin{tabular}{|c|c|c|c|c|c|c|c|c|}
\hline Correlations $^{a}$ & & & $\begin{array}{l}\text { Lateral position } \\
\text { of the glenoid } \\
\text { fossa@T0 }\end{array}$ & $\begin{array}{l}\text { Lateral position } \\
\text { of the condyles } \\
\text { @ T0 }\end{array}$ & $\begin{array}{l}\text { Nasal } \\
\text { width@ } \\
\text { T0 }\end{array}$ & $\begin{array}{l}\text { Lateral position of } \\
\text { the glenoid fossa } \\
\text { @ T2 }\end{array}$ & $\begin{array}{l}\text { Lateral } \\
\text { position of the } \\
\text { condyles@T2 }\end{array}$ & $\begin{array}{l}\text { Nasal } \\
\text { width@ } \\
\text { T2 }\end{array}$ \\
\hline \multirow{4}{*}{$\begin{array}{l}\text { Lateral position } \\
\text { of the glenoid } \\
\text { fossa @T0 }\end{array}$} & \multirow{2}{*}{\multicolumn{2}{|c|}{$\begin{array}{l}\text { Pearson correlation } \\
p \text { value }\end{array}$}} & \multirow[t]{4}{*}{1} & $0.821^{* *}$ & 0.330 & $0.952^{* *}$ & $0.835^{* *}$ & 0.284 \\
\hline & & & & 0.000 & 0.107 & 0.000 & 0.000 & 0.169 \\
\hline & \multirow{2}{*}{$\begin{array}{l}\text { BCa 95\% confidence } \\
\text { interval }\end{array}$} & Lower & & 0.595 & -0.068 & 0.885 & 0.684 & -0.062 \\
\hline & & Upper & & 0.941 & 0.628 & 0.988 & 0.926 & 0.582 \\
\hline \multirow{4}{*}{$\begin{array}{l}\text { Lateral position } \\
\text { of the condyles } \\
@ \text { T0 }\end{array}$} & \multirow{2}{*}{\multicolumn{2}{|c|}{$\begin{array}{l}\text { Pearson correlation } \\
p \text { value }\end{array}$}} & $0.821^{* *}$ & 1 & 0.282 & $0.815^{* *}$ & $0.964^{* *}$ & 0.167 \\
\hline & & & 0.000 & & 0.172 & 0.000 & 0.000 & 0.425 \\
\hline & \multirow{2}{*}{$\begin{array}{l}\text { BCa 95\% confidence } \\
\text { interval }\end{array}$} & Lower & 0.595 & & -0.140 & 0.557 & 0.920 & -0.142 \\
\hline & & Upper & 0.941 & & 0.663 & 0.935 & 0.983 & 0.457 \\
\hline \multirow{4}{*}{$\begin{array}{l}\text { Nasal width } \\
@ \text { T0 }\end{array}$} & \multirow{2}{*}{\multicolumn{2}{|c|}{$\begin{array}{l}\text { Pearson correlation } \\
p \text { value }\end{array}$}} & 0.330 & 0.282 & 1 & 0.372 & $0.398^{*}$ & $0.839^{* *}$ \\
\hline & & & 0.107 & 0.172 & & 0.067 & 0.049 & 0.000 \\
\hline & BCa $95 \%$ confidence & Lower & -0.068 & -0.140 & & 0.014 & 0.052 & 0.610 \\
\hline & interval & Upper & 0.628 & 0.663 & & 0.641 & 0.686 & 0.950 \\
\hline \multirow{4}{*}{$\begin{array}{l}\text { Lateral position } \\
\text { of the glenoid } \\
\text { fossa @ T2 }\end{array}$} & \multirow{2}{*}{\multicolumn{2}{|c|}{$\begin{array}{l}\text { Pearson correlation } \\
p \text { value }\end{array}$}} & $0.952^{* *}$ & $0.815^{* *}$ & 0.372 & 1 & $0.829^{* *}$ & 0.374 \\
\hline & & & 0.000 & 0.000 & 0.067 & & 0.000 & 0.065 \\
\hline & \multirow{2}{*}{$\begin{array}{l}\text { BCa 95\% confidence } \\
\text { interval }\end{array}$} & Lower & 0.885 & 0.557 & 0.014 & & 0.631 & 0.061 \\
\hline & & Upper & 0.988 & 0.935 & 0.641 & & 0.944 & 0.618 \\
\hline \multirow{4}{*}{$\begin{array}{l}\text { Lateral position } \\
\text { of the condyles } \\
\text { @ T2 }\end{array}$} & \multirow{2}{*}{\multicolumn{2}{|c|}{$\begin{array}{l}\text { Pearson correlation } \\
p \text { value }\end{array}$}} & $0.835^{* *}$ & $0.964^{* *}$ & $0.398^{*}$ & $0.829 * *$ & 1 & 0.296 \\
\hline & & & 0.000 & 0.000 & 0.049 & 0.000 & & 0.151 \\
\hline & \multirow{2}{*}{$\begin{array}{l}\text { BCa 95\% confidence } \\
\text { interval }\end{array}$} & Lower & 0.684 & 0.920 & 0.052 & 0.631 & & -0.031 \\
\hline & & Upper & 0.926 & 0.983 & 0.686 & 0.944 & & 0.555 \\
\hline \multirow{4}{*}{$\begin{array}{l}\text { Nasal width } \\
@ \text { T2 }\end{array}$} & \multirow{2}{*}{\multicolumn{2}{|c|}{$\begin{array}{l}\text { Pearson correlation } \\
p \text { value }\end{array}$}} & 0.284 & 0.167 & $0.839^{* *}$ & 0.374 & 0.296 & 1 \\
\hline & & & 0.169 & 0.425 & 0.000 & 0.065 & 0.151 & \\
\hline & \multirow{2}{*}{$\begin{array}{l}\text { BCa 95\% confidence } \\
\text { interval }\end{array}$} & Lower & -0.062 & -0.142 & 0.610 & 0.061 & -0.031 & \\
\hline & & Upper & 0.582 & 0.457 & 0.950 & 0.618 & 0.555 & \\
\hline
\end{tabular}

BCa: Bias corrected accelerated

${ }^{*}$ Correlation is significant at the 0.05 level (two-tailed)

${ }^{* *}$ Correlation is significant at the 0.01 level (two-tailed)

aUnless otherwise noted, bootstrap results are based on 1000 bootstrap samples

At T0, the lateral position of the glenoid fossa was highly correlated with the lateral position of the condyles, but its correlation with the nasal cavity width did not reach statistical significance. Similarly, at T0, the lateral position of the condyles was not correlated with the nasal cavity width (Fig. 5).

At T2, the lateral position of the glenoid fossa was highly correlated with the lateral position of the condyles, with the same magnitude as T0, and its correlation with the nasal cavity width was close to statistical significance. Additionally, at T2, the lateral position of the condyles was not correlated with the nasal cavity width (Fig. 6).

\section{Discussion}

Dentofacial orthopedic treatment, particularly RME, is indicated for a wide variety of clinical conditions routinely faced by the orthodontist. $^{8}$ In youngsters, morphological problems involve frequently an underdevelopment of the midface, which can be expressed by a constricted high-arched palate and reduced transverse and/or sagittal maxillary growth.,10

Maxillary constriction can be associated with several problems that include esthetics and occlusal disharmony such as posterior crossbite the persistence of which can instigate permanent 


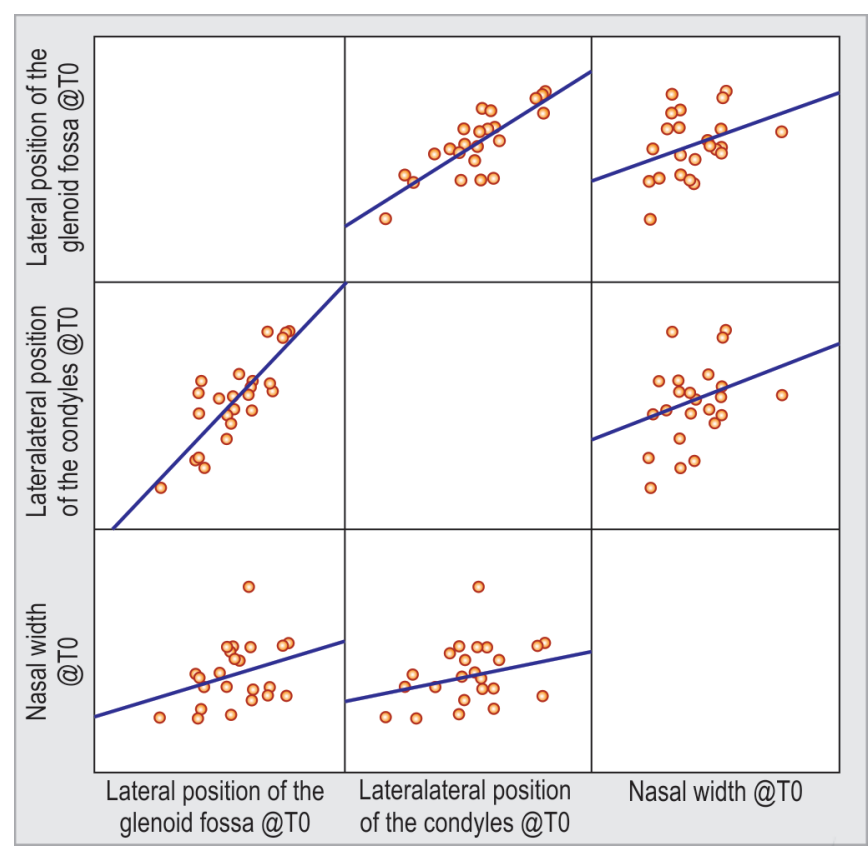

Fig. 5: Correlation between the predefined CBCT parameters at TO

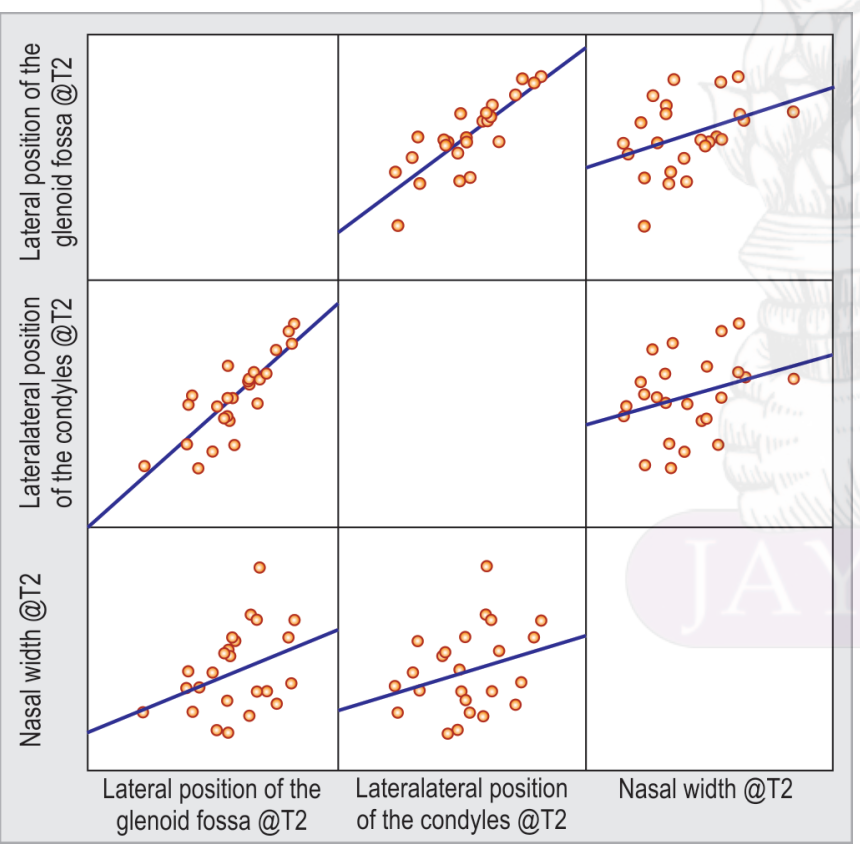

Fig. 6: Correlation between the predefined CBCT parameters at $\mathrm{T} 2$

changes in the bony base and probably in the growth center at the temporomandibular joint. ${ }^{5}$ It has been demonstrated that malocclusions, especially transverse anomalies, have an obvious effect on mandibular condyle morphology. ${ }^{11-14}$

Baratieri et al. in a study using CBCT scans to assess the transverse effects on the nasomaxillary complex in patients subjected to RME in comparison to untreated individuals showed a stable increase in molar, maxillary, palatal, and nasal transverse dimensions in comparison to the control group 1 year after treatment with RME. ${ }^{15}$ Early orthopedic treatment with RME also is beneficial in avoiding the development of facial skeletal asymmetry that may lead to both functional and structural imbalances in growing patients. If left uncorrected, such a functional shift may lead to the development of temporomandibular disorders and other related conditions later in life. ${ }^{5}$

These papers showed the relationship between the transverse malocclusion and the TMJ dysfunction. Consequently, the current study strived to test the correlation between the two major components of these joints (glenoid fossas and mandibular condyles) together and with the nasal cavity width. The patients treated in this study displayed a true transverse skeletal discrepancy with a bilateral crossbite which required an orthopedic treatment with RME.

For several decades, two-dimensional (2D) cephalometric radiographs have been used successfully in orthodontics to diagnose and treat these three-dimensional (3D) malocclusions. Despite its efficiency, it does present some shortcomings and limitations. In fact, these radiographs are subject to projection errors as well as landmark identification and measurement problems because they can only produce 2D data from a 3D object. ${ }^{16,17}$ In comparison, 3D volumetric imaging, such as $\mathrm{CBCT}$, presents a better geometric precision and spatial resolution than the conventional computerized one. ${ }^{18}$ Moreover, $\mathrm{CBCT}$ measurements are not significantly affected by variation in skull orientation or the head position during image acquisition. ${ }^{19,20}$ Hence, the present study was based on comparison of data before and after expansion on $\mathrm{CBCT}$ images to acquire reliable and accurate outcomes. In fact, the SEDENTEXCT guidelines stated that in the generalized application of CBCT for the developing dentition, studies of measurement accuracy are highly relevant in orthodontic diagnosis and treatment planning and advocate that $\mathrm{CBCT}$ can produce precise depictions of tooth inter-relationships and associated bony anatomy. ${ }^{21}$

This research tested the correlation after RME in 25 growing patients aged $11.6 \pm 1.6$ years between three variates involving the nasal cavity width, interglenoid fossa distance and intercondylar distance at T0 (baseline) and T2 (6 months after the end of expander activation), seeking two objectives:

- The condyle-fossa adaptation at the TMJs after RME to evaluate the correlation between the interglenoid fossa distance and the intercondylar distance.

- The correlation between the transverse variation of the nasal cavity width, structure adjacent to the expansion site, and more distant ones involving interglenoid fossa distance and intercondylar distance.

Few studies have investigated the impact of RME on the condyle fossa relationship; ${ }^{22,23}$ inversely, there is abundant literature on its effect on the nasal width; ${ }^{24-27}$ and no correlation was inspected to date between these important maxillofacial components involved in major oral functions including respiration, mastication, and TMJ operation.

The results showed that at $\mathrm{TO}$, the lateral position of the glenoid fossa was already highly correlated with the lateral position of the mandibular condyles and this correlation remained highly significant after RME, showing that the transverse adaptation was relevant at $\mathrm{T} 2$ and that there was no appearance of any anatomical asymmetries.

Similarly, McLeod et al. showed that RME treatments with a toothanchored appliance are not associated with a significant condylar positional change when evaluated at 6 months in comparison to a control group. ${ }^{6}$ Another study conducted by Melgaço et al. concluded that RME induces immediate changes in the position of the condyles in patients with maxillary constriction without posterior crossbite, and no condylar asymmetries were observed when comparing the 
right and left sides. No correlation was noticed in both studies, but no contraindication regarding the use of RME was notified. ${ }^{2}$

For the correlation between the nasal cavity width and interglenoid fossa distance, results revealed close to significant values ( $p=0.065$ ) at T2. Besides, the correlation between the nasal cavity width and intercondylar distance was not significant at T2 $(p=0.151)$. The interpretation of outcomes might suggest that the glenoid fossas, part of the temporal bone, could be affected by the expansion. Inversely, the condyles, components of a mobile bone of the mandible, are less affected by RME. In fact, Kilic et al. revealed that RME has an influence on structures in the temporal bone localized relatively distant from the site of activation and neighboring the glenoid fossa. ${ }^{28,29}$ In a study to evaluate the stress distribution and displacement during an intermaxillary disjunction, a finite element model of a dry human male skull was produced from CT scans. The results determined that the highest stress was observed in the maxilla and extents throughout almost the whole frontal skull structures. The most affected regions presenting a high compressive stress were observed to have an important and active cellular activity. The temporal bone demonstrated a substantial displacement in the transverse dimension. ${ }^{30}$

\section{Limitations of the Study}

In this research, only linear measurements were taken automatically thanks to the software used. More studies are needed to reveal the remodeling at the TMJs using color map superimpositions as in the study of De Clerck et al. where three-dimensional changes were demonstrated in the position of the mandible and a remodeling in the temporal fossa following the use of class III intermaxillary elastics hooked on bilateral miniplates. ${ }^{31}$

\section{Conclusions}

The current study shows that high transverse correlation exists between the interglenoid fossa distance and intercondylar one after RME. A close-to-significant correlation was demonstrated for the nasal width and interglenoid fossa distance, and there was no correlation between the nasal width and the intercondylar distance. Further investigations are needed using 3D regional color map superimpositions.

\section{Declarations}

\section{Ethics Approval and Consent to Participate}

This protocol has been approved by the Research Ethics Committee of Dental School, Lebanese University (Decision No. 31.04.2015). Informed consent was obtained from children's parents or guardians before participation in the study. Participants' confidentiality was ensured using identification code numbers and the information available only to researchers.

\section{Availability of Data and Material}

The datasets generated and/or analyzed during the current study will be made available from the corresponding author on reasonable request.

\section{Clinical Significance}

The outcomes showed a consistent condyle fossa adaptability at the temporomandibular joints (TMJs) in youngsters after RME.

\section{ACKnOWLedgments}

The protocol was approved by the Ethics Committee of the Lebanese University, National Institute in Lebanon [CUEMB Process Number 31/04/2015]. The study was funded by the Lebanese University and Centre National de Recherche Scientifique (CNRS), Lebanon [Number: 652 on 14/04/2016]. The CNRS has no role in the design of the study, neither collection, analysis, nor interpretation of data or in writing the manuscript.

\section{References}

1. Haas AJ. Palatal expansion: just the beginning of dentofacial orthopedics. Am J Orthod 1970;57(3):219-255.

2. Melgaço CA, Columbano Neto J, et al. Immediate changes in condylar position after rapid maxillary expansion. Am J Orthod Dentofacial Orthop 2014;145(6):771-779. DOI: 10.1016/j. ajodo.2014.01.024.

3. Haas AJ. The Treatment of Maxillary Deficiency by Opening the Midpalatal Suture. Angle Orthod 1965;35:200-217. DOI: 10.1043/0003-3219(1965)035<0200:TTOMDB>2.0.CO;2.

4. Angelieri $F$, Franchi $L$, et al. Prediction of rapid maxillary expansion by assessing the maturation of the midpalatal suture on cone beam CT. Dental Press J Orthod 2016;21(6):115-125. DOI: 10.1590/21776709.21.6.115-125.sar.

5. McNamara Jr JA, Lione R, et al. The role of rapid maxillary expansion in the promotion of oral and general health. Prog Orthod 2015;16-33. DOI: 10.1186/s40510-015-0105-x.

6. McLeod L, Hernandez IA, et al. Condylar positional changes in rapid maxillary expansion assessed with cone-beam computer tomography. Int Orthod 2016;14(3):342-356. DOI: 10.1016/j. ortho.2016.07.006.

7. Sayegh-Ghoussoub M. Effect of rapid maxillary expansion on glenoid fossa and condylar position in growing patients. http://isrctn.com/ [Internet]. Springer Nature; 2017 Nov 7.

8. McNamara JA. Maxillary transverse deficiency. Am J Orthod Dentofacial Orthop 2000;117(5):567-570.

9. Lione R, Ballanti F, et al. Treatment and posttreatment skeletal effects of rapid maxillary expansion studied with low-dose computed tomography in growing subjects. Am J Orthod Dentofacial Orthop 2008;134(3):389-392. DOI: 10.1016/j.ajodo.2008.05.011.

10. Eichenberger $M$, Baumgartner $S$. The impact of rapid palatal expansion on children's general health: a literature review. Eur J Paediatr Dent 2014;15(1):67-71.

11. Piancino MG, Talpone $F$, et al. Reverse-sequencing chewing patterns before and after treatment of children with a unilateral posterior crossbite. Eur J Orthod 2006;28(5):480-484. DOI: 10.1093/ ejo/cjl014.

12. Tadej G, Engstrom C, et al. Mandibular condyle morphology in relation to malocclusions in children. Angle Orthod 1989;59(3):187-194. DOI: 10.1043/0003-3219(1989)059<0187:MCMIRT>2.0.CO;2.

13. Solberg WK, Bibb CA, et al. Malocclusion associated with temporomandibular joint changes in young adults at autopsy. Am J Orthod 1986;89(4):326-330.

14. Kusayama M, Motohashi N, et al. Relationship between transverse dental anomalies and skeletal asymmetry. Am J Orthod Dentofacial Orthop 2003;123(3):329-337. DOI: 10.1067/mod.2003.41.

15. Baratieri C, Alves Jr M, et al. 3D mandibular positioning after rapid maxillary expansion in class II malocclusion. Braz Dent J 2011;22(5):428-434.

16. Lagravere MO, Gordon JM, et al. Reliability of traditional cephalometric landmarks as seen in three-dimensional analysis in maxillary expansion treatments. Angle Orthod 2009;79(6):1047-1056. DOI: 10.2319/010509-10R.1.

17. Lagravere MO, Low $\mathrm{C}$, et al. Intraexaminer and interexaminer reliabilities of landmark identification on digitized lateral cephalograms and formatted 3-dimensional cone-beam computerized tomography 
images. Am J Orthod Dentofacial Orthop 2010;137(5):598-604. DOI: 10.1016/j.ajodo.2008.07.018.

18. Cevidanes LH, Bailey LJ, et al. Three-dimensional cone-beam computed tomography for assessment of mandibular changes after orthognathic surgery. Am J Orthod Dentofacial Orthop 2007;131(1):44-50. DOI: 10.1016/j.ajodo.2005.03.029.

19. Ludlow JB, Laster WS, et al. Accuracy of measurements of mandibular anatomy in cone beam computed tomography images. Oral Surg Oral Med Oral Pathol Oral Radiol Endod 2007;103(4):534-542. DOI: 10.1016/j.tripleo.2006.04.008.

20. Frongia G, Piancino MG, et al. Cone-beam computed tomography: accuracy of three-dimensional cephalometry analysis and influence of patient scanning position. J Craniofac Surg 2012;23(4):1038-1043. DOI: 10.1097/SCS.0b013e318252d5e1.

21. Commission E. Cone beam CT for dental and maxillofacial radiologyevidence-based guidelines. (Euratom) TSFPotEAEC; 2012.

22. Kecik D, Kocadereli I, et al. Evaluation of the treatment changes of functional posterior crossbite in the mixed dentition. Am J Orthod Dentofacial Orthop 2007;131(2):202-215.DOI: 10.1016/j.ajodo.2005.03.030.

23. Arat FE, Arat ZM, et al. Muscular and condylar response to rapid maxillary expansion. Part 2: magnetic resonance imaging study of the temporomandibular joint. Am J Orthod Dentofacial Orthop 2008;133(6):823-829. DOI: 10.1016/j.ajodo.2006.07.029.

24. Di Carlo G, Saccucci M, et al. Rapid maxillary expansion and upper airway morphology: a systematic review on the role of cone beam computed tomography. Biomed Res Int 2017;2017:5460429. DOI: $10.1155 / 2017 / 5460429$.
25. El H, Palomo JM. Three-dimensional evaluation of upper airway following rapid maxillary expansion: a CBCT study. Angle Orthod 2014;84(2):265-273. DOI: 10.2319/012313-71.1.

26. Ribeiro AN, de Paiva JB, et al. Upper airway expansion after rapid maxillary expansion evaluated with cone beam computed tomography. Angle Orthod 2012;82(3):458-463. DOI: 10.2319/ 030411-157.1.

27. Cordasco G, Nucera R, et al. Effects of orthopedic maxillary expansion on nasal cavity size in growing subjects: a low dose computer tomography clinical trial. Int J Pediatr Otorhinolaryngol 2012;76(11):1547-1551. DOI: 10.1016/j.ijporl.2012.07.008.

28. Kilic $\mathrm{N}$, Oktay $\mathrm{H}$. Effects of rapid maxillary expansion on nasal breathing and some naso-respiratory and breathing problems in growing children: a literature review. Int J Pediatr Otorhinolaryngol 2008;72(11):1595-1601. DOI: 10.1016/j.jijporl.2008.07.014.

29. Kilic N, Yoruk O, et al. Rapid maxillary expansion versus middle ear tube placement: comparison of hearing improvements in children with resistance otitis media with effusion. Angle Orthod 2016;86(5):761-767. DOI: 10.2319/101515-693.1.

30. Boryor A, Geiger M, et al. Stress distribution and displacement analysis during an intermaxillary disjunction-a three-dimensional FEM study of a human skull. J Biomech 2008;41(2):376-382. DOI: 10.1016/j. jbiomech.2007.08.016.

31. De Clerck H, Nguyen T, et al. Three-dimensional assessment of mandibular and glenoid fossa changes after bone-anchored class III intermaxillary traction. Am J Orthod Dentofacial Orthop 2012;142(1):25-31. DOI: 10.1016/j.ajodo.2012.01.017. 